\title{
BAYESIAN DETECTION OF SIGNAL UNDER RAYLEIGH MULTIPLICATIVE NOISE BASED ON REVERSIBLE JUMP MCMC
}

\author{
*Suparman ${ }^{1}$, Mohammad Toifur ${ }^{2}$, Asnul Dahar Minghat ${ }^{3}$, Eviana Hikamudin $^{4}$, and Mohd Saifullah Rusiman ${ }^{5}$ \\ ${ }^{1,2}$ Faculty of Teacher Training and Education, Universitas Ahmad Dahlan, Indonesia \\ ${ }^{3}$ Razak Faculty of Technology and Informatics, Universiti Teknologi Malaysia, Malaysia \\ ${ }^{4}$ Center for Educational Assessment, Kementerian Pendidikan Kebudayaan Riset dan Teknologi, Indonesia \\ ${ }^{5}$ Faculty of Applied Sciences and Technology, Universiti Tun Hussein Onn Malaysia, Malaysia
}

* Corresponding Author, Received: 06 Oct. 2021, Revised: 01 Dec. 2021, Accepted: 09 Jan. 2022

\begin{abstract}
Piecewise constant models have been used in signal processing. The signal contains noise so that noise needs to be eliminated. Several research results have used the assumption that noise has a normal, gamma, or laplace distribution. However, the signal may have noise with other distributions. This study aims to propose a piecewise constant model in which noise is assumed to have a Rayleigh distribution. This study also proposes a method for estimating the parameters of a piecewise constant model that contains Rayleigh noise. The parameters of the piecewise constant model were estimated in the Bayesian framework by adopting the reversible jump Markov Chain Monte Carlo (MCMC) method. This research shows that the dimension of the parameter space is a combination of several spaces with different dimensions. Bayes estimators for the parameters of the piecewise constant model cannot be stated explicitly. The reversible jump MCMC method is used to calculate the Bayes estimator. The results of this study have a significant contribution in providing Rayleigh noise as an alternative noise in signal processing. This research has a novelty, namely: the use of Rayleigh noise in the piecewise constant model and the hierarchical Bayesian procedure to estimate the parameters of the piecewise constant model. Further research can be extended to the estimation procedure of the piecewise constant with Weibull noise.
\end{abstract}

Keywords: Bayesian, Piecewise constant, Rayleigh noise, Reversible jump MCMC, Signal detection

\section{INTRODUCTION}

Piecewise is a stochastic mathematical model. The piecewise models are widely used in changepoint analysis, for example [1-3]. One of the piecewise models used in signal processing is the piecewise constant model. The piecewise constant model contains a noise. Several authors use additive noise, for example [2,4]. Several other authors use multiplicative noise, for example [5-7]. Noise is usually assumed to follow a distribution. Some researchers use the piecewise constant model with Gamma noise, for example [5-7]. In [5], the value of the Gamma distribution parameter is fixed.

In applications, the value of this parameter is generally unknown. In [6,7], the value of one parameter is fixed and the value of the other parameter is assumed to be unknown. This parameter is estimated based on the data. However, in various applications of the piecewise constant model, the noise of the mathematical model is not Gamma distributed. There is little literature on piecewise constant models with multiplicative noise that is not a gamma distribution. This article provides a solution by proposing a piecewise constant model with Rayleigh noise. In this article, noise is assumed to have a Rayleigh distribution. The use of Rayleigh noise in piecewise constant is an innovation because the piecewise constant model with Rayleigh noise has not been studied by previous researchers. However, the piecewise constant model has a complex structure because the number of constant models is also a parameter. This article provides a solution by proposing a reversible jump Markov Chain Monte Carlo (MCMC) algorithm to estimate the parameters of the piecewise constant model.

This article aims to find the procedure for estimating the piecewise constant model with Rayleigh noise based on reversible jump MCMC algorithm.

\section{RESEARCH SIGNIFICANCE}

The significance of the study is the use of Rayleigh noise in the piecewise constant model and the hierarchical Bayesian procedure to estimate the parameters of the piecewise constant model.

\section{LITERATUR REVIEW}

\subsection{Rayleigh Distribution}

Suppose that $z$ is a random variable. The random variable $z$ is said to have a Rayleigh distribution with parameter $\sigma$, if the probability 
density function of $z$ is [8]

$$
g(z \mid \sigma)=\frac{z}{\sigma^{2}} \exp -\frac{z^{2}}{2 \sigma^{2}}
$$

\subsection{Piecewise Constant Model with Multiplicative Noise}

Let $y=\left(\mathrm{y}_{1}, \cdots, \mathrm{y}_{\mathrm{n}}\right)$ be a data set where $\mathrm{n}$ is the number of observations. The data is said to have a piecewise constant model with multiplicative noise if this data satisfies the stochastic equation

$$
y_{t}=\mu_{t} z_{t}
$$

where $\mu_{t}$ is a constant function [9]:

$$
\mu_{t}=\left\{\begin{array}{cc}
h_{1}, & \tau_{1}<t \leq \tau_{2} \\
h_{2}, & \tau_{2}<t \leq \tau_{3} \\
\cdots & \cdots \\
h_{k+1}, & \tau_{k+1}<t \leq \tau_{k+2}
\end{array}\right.
$$

where $\tau_{1}=0$ and $\tau_{k+2}=n$. Here, $z, \ldots, z_{n}$ are noise and $k$ is the number of change-point. The quantity $\tau=\left(\tau, \ldots, \tau_{k}\right)$ is the location of the change-point. The quantity $h=\left(h, \ldots, h_{k+1}\right)$ is the height of the constant function. The piecewise constant model with multiplicative noise is used in modeling the Synthetic Aperture Radar (SAR) signal [10]. Research related to SAR signal can also be found in [5-7,11]. In [5-7], the noise is assumed to have a Gamma distribution. In this article, the noise $z_{t}$ is assumed to have a Rayleigh distribution with the parameter $\sigma$.

\subsection{Bayesian Approach}

Let $\theta=(k, \tau, h, \sigma)$ be the parameter of the piecewise constant model. As in [12], the parameter $\theta$ was estimated using the Bayesian approach. In the Bayesian approach, the parameter $\theta$ is considered as a random variable that has a prior distribution. The prior distribution of parameter $\theta$ is combined with the likelihood function of $y_{t}$ $(t=1,2, \cdots, n)$ to produce the posterior distribution of parameter $\theta$. Since the number of change-point $k$ is also a parameter, the parameter space of $\theta$ is a combination of $\mathrm{k}$ parameter spaces with different dimensions. Therefore, the posterior distribution of the parameter $\theta$ has a complex structure. So the Bayes estimator of the parameter $\theta$ cannot be calculated explicitly. To solve this problem, the Bayesian estimator is calculated using the reversible jump MCMC algorithm.

\subsection{Reversible Jump MCMC}

The reversible jump MCMC method proposed by Green [13]. The reversible jump MCMC method is an extension of the standard MCMC method. In the standard MCMC, the simulation of the posterior distribution is only in spaces with the same dimensions. In the reversible jump MCMC, Markov chains can move from one space to another space with different dimensions. In this article, the reversible jump MCMC is used to calculated the Bayes estimator of the parameter $\theta$.

The basic idea of the reversible jump MCMC is to create a Markov chain that has a limit distribution close to the posterior distribution. This Markov chain is used to determine the estimator of the parameter $\theta$. For example, this Markov chain is used to determine the Posterior Marginal Maximum estimator [14].

\section{METHODOLOGY}

The data used in this article is the data that will be modeled with the piecewise constant model. Procedures for change-point detection include data collection, data modeling, and estimation of model parameters. The steps in estimating the model parameters consist of selecting the prior distribution, determining the likelihood function, determining the posterior distribution, and calculating the Bayes estimator. The Bayes estimator was determined using the reversible jump MCMC algorithm.

\section{RESULTS AND DISCUSSION}

\subsection{Likelihood Function}

Let $y=\left(y_{1}, \ldots, y_{n}\right)$ be data. This data is assumed to have a piecewise constant model with multiplicative noise. In this article, the noise $z_{t}$ is assumed to have a Rayleigh distribution with the parameter $\beta$ where $\beta=\frac{1}{\sigma^{2}}$. Therefore the probability function of $z_{t}$ can be written as

$$
g\left(z_{t} \mid \beta\right)=\beta z_{t} \exp -\frac{1}{2} \beta z_{t}^{2}
$$

So the likelihood function of data $y$ becomes

$$
\begin{aligned}
& f(y \mid k, \tau, h, \beta) \\
& \quad=\beta^{n} \prod_{i=1}^{k+1}\left(\prod_{t=\tau_{-} i+1}^{\tau_{i+1}} y_{t}\right) \frac{1}{h_{i}^{2 n_{i}}} \exp -\beta \frac{s_{i}}{h_{i}^{2}}
\end{aligned}
$$

where $s_{i}=\frac{1}{2} \sum_{t=\tau_{i}+1}^{\tau_{i+1}} y_{t}^{2} \quad$ and $\quad n_{i}=\tau_{i+1}-\tau_{i}$ for $i=1, \ldots, k+1$.

\subsection{Prior Distribution}

As in [6-7], the prior distribution of the number of change-points $k$ is Binomial distribution with parameters $k_{\max }$ and $\lambda$. Here, $k_{\max }$ is the 
maximum value of $k$. While the prior distribution of $\lambda$ is a uniform distribution on the interval $(0,1)$. As in [13], the prior distribution of change-point locations $\tau_{1}, \ldots, \tau_{k}$ is an ordered statistic. Here, the prior distribution of height $h=\left(h_{1}, \ldots ., h_{k+1}\right)$ is chosen so that the prior distribution of $h_{1}^{2}, \ldots, h_{k+1}^{2}$ is an inverse exponential distribution with parameter $v>0$. Therefore, the probability function of $h_{1}^{2}, \ldots, h_{k+1}^{2}$ can be written as

$$
\begin{aligned}
& \pi(h \mid k, v) \\
& =v^{(k+1)} \prod_{i=1}^{k+1}\left(h_{i}^{2}\right)^{-2} \exp -v \sum_{i=1}^{k+1} \frac{1}{h_{i}^{2}} .
\end{aligned}
$$

While the prior distribution of $v$ is the Jeffreys distribution. The prior distribution of $\beta$ is also the Jeffreys distribution. Finally, the joint prior distribution of $\theta=(k, \tau, h, \lambda, v, \beta)$ becomes

$$
\begin{aligned}
& \pi(k, \tau, h, \lambda, v, \beta) \\
&=C_{k}^{k_{\max }} \lambda^{k}(1-\lambda)^{k_{\max }-k} \frac{1}{C_{n-2}^{2 k+1}} \\
& \prod_{i=1}^{k+1}\left(n_{i}-1\right) v^{(k+1)} \prod_{i=1}^{k+1}\left(h_{i}^{2}\right)^{-2} \\
& \exp -v \sum_{i=1}^{k+1} \frac{1}{h_{i}^{2}} \frac{1}{\beta} \frac{1}{v} .
\end{aligned}
$$

\subsection{Posterior Distribution}

Let $\theta=\left(\theta_{1}, \theta_{2}\right)$ be a parameter. Here, $\theta_{1}=$ $(k, \tau, h)$ and $\theta_{2}=(\lambda, v, \beta)$. According to Bayes Theorem, the posterior distribution of the parameter $\theta=\left(\theta_{1}, \theta_{2}\right)$ is

$$
\begin{gathered}
\pi\left(\theta_{1}, \theta_{2} \mid y\right) \\
\propto \frac{\beta^{\alpha n-1}}{(\Gamma(\alpha))^{n}} \prod_{i=1}^{k+1} y_{t}^{n_{i}} \frac{1}{h_{i}^{2 n_{i}}} \exp -\beta \frac{s_{i}}{h_{i}^{2}} C_{k}^{k_{\max }} \\
\lambda^{k}(1-\lambda)^{k_{\max }-k} \prod_{i=1}^{k+1}\left(n_{i}-1\right) \\
v^{k} \prod_{i=1}^{k+1}\left(h_{i}^{2}\right)^{-2} \exp -v \sum_{i=1}^{k+1} \frac{1}{h_{i}^{2}}
\end{gathered}
$$

\subsection{Reversible Jump MCMC}

As in [6-7], the algorithm for estimating the parameter $\left(\theta_{1}, \theta_{2}\right)$ is as follows. First, simulate the distribution $\pi\left(\theta_{2} \mid \theta_{1}, y\right)$. This simulation consists of three steps, namely:

$$
\begin{aligned}
& \beta \sim G\left(2 n, \sum_{i=1}^{k+1} \frac{s_{i}}{h_{i}}\right), \\
& \lambda \sim B\left(k+1, k_{\max }-k+1\right), \text { and } \\
& v \sim G\left(k+1, \sum_{i=1}^{k+1} \frac{1}{h_{i}^{2}}\right) .
\end{aligned}
$$

Second, simulate the distribution $\pi\left(\theta_{1} \mid \theta_{2}, y\right)$. The distribution $\pi\left(\theta_{1} \mid \theta_{2}, y\right)$ is simulated using the reversible jump MCMC algorithm. As in [7], the reversible jump MCMC algorithm uses 3 transformations, namely: change-point location shift, change-point birth, and change-point death. These transformations have been used in [7] so that more detailed calculations in [7] can be used but Gamma noise is replaced by Rayleigh noise. Although the calculations in [7] and the calculations in this article are similar, they are conceptually different.

\subsection{Validation}

In this article, the performance of the reversible jump MCMC algorithm is validated using synthetic data. First, the model parameter values are selected. Then, the data synthesis is made based on this model. After that, the MCMC reversible jump algorithm is run where the input of the algorithm is this synthesis data. The output of the algorithm is a parameter estimator of the model. Finally, the parameter estimators of the model are compared with the parameter values of the previously selected model. The reversible jump MCMC algorithm is categorized as valid if the parameter estimator value of the model generated by the algorithm is close to the parameter value of the previously determined model.

The first synthesis data, the synthesis data is created using the parameter values in Table 1 (second row). Meanwhile, the parameter values for the second and third synthesis data are given in the third and fourth rows of Table 1 . These synthesis data are presented in Fig. 1, Fig. 4, and Fig. 7.

Table 1 The parameter values of the synthesis data

\begin{tabular}{ccc}
\hline$k$ & $\tau$ & $h$ \\
\hline 4 & $(1,80,120,170,200,250)$ & $(1.0,1.6,0.8,0.2,0.7)$ \\
5 & $(1,40,80,120,170,200,250)$ & $(1.5,1.1,1.6,0.8,0.4,0.7)$ \\
6 & $(1,30,80,120,170,200,250,350)$ & $(1.0,0.8,1.5,0.7,1.5,0.4,1.0)$ \\
\hline
\end{tabular}




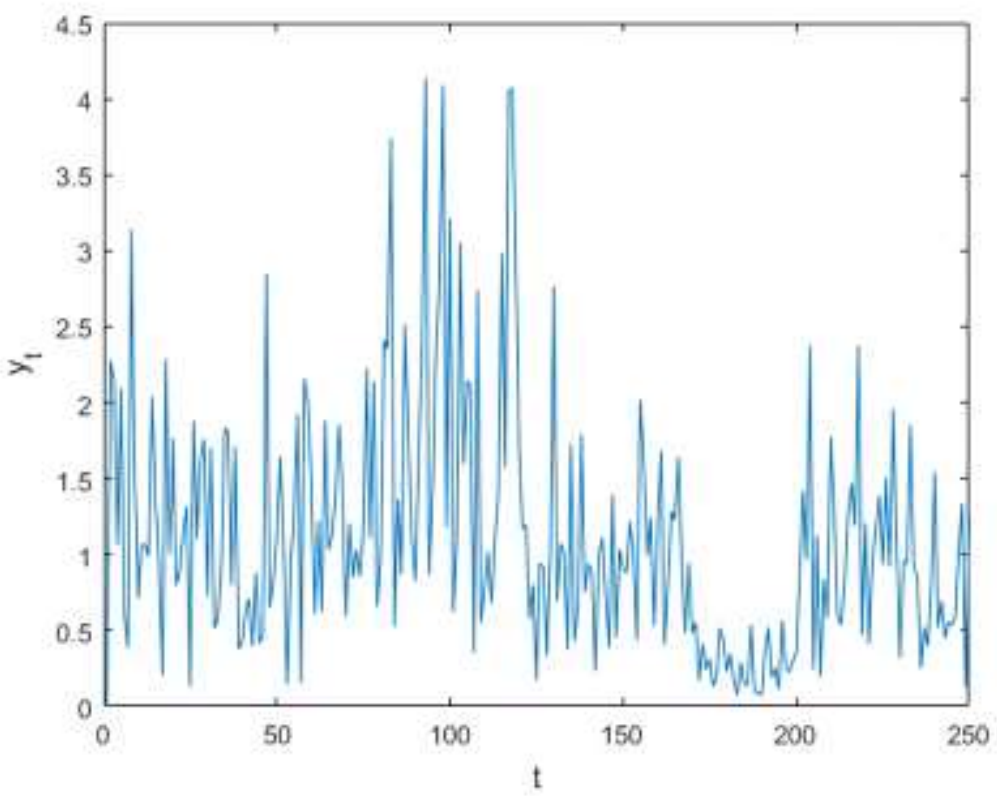

Fig. 1 Synthesis data with 4 change points

With this synthesis data as input, the reversible jump MCMC algorithm is run for 100000 iterations and a burn-in period of 25000. The output of the algorithm is an estimator of model parameters. The histogram of the number of change-points is presented in Fig. 2. Meanwhile, estimators of other model parameters are presented in Table 2 (second row). The superposition between the first synthesis data and the parameter estimator is presented in Fig. 3. This method was repeated for the second synthesis data and the third synthesis data. The model parameter estimates for the second and third synthesis data are presented in Figs. 4-9.

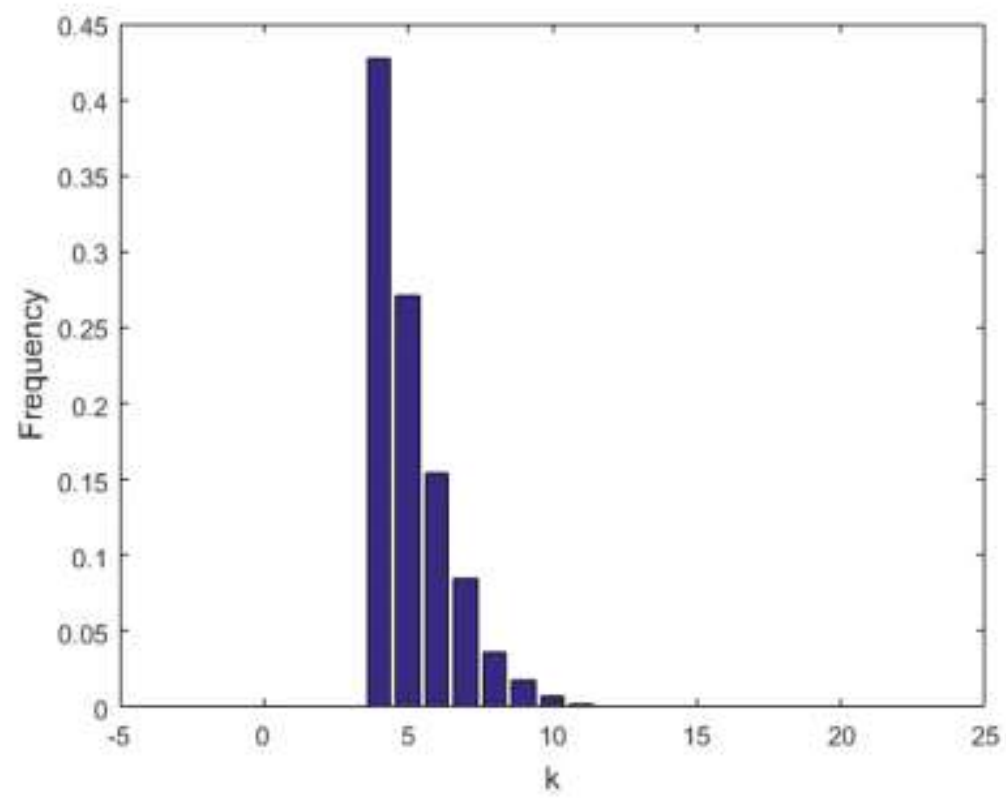

Fig. 2 Histogram of the number of change-points (first synthesis data) 


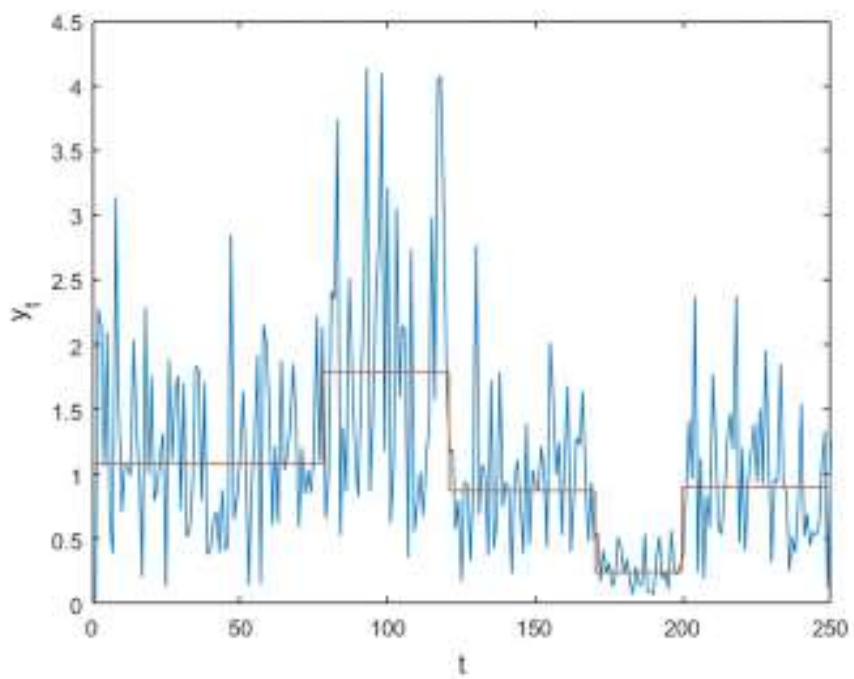

Fig. 3 Superposition between first synthesis data and parameter estimator

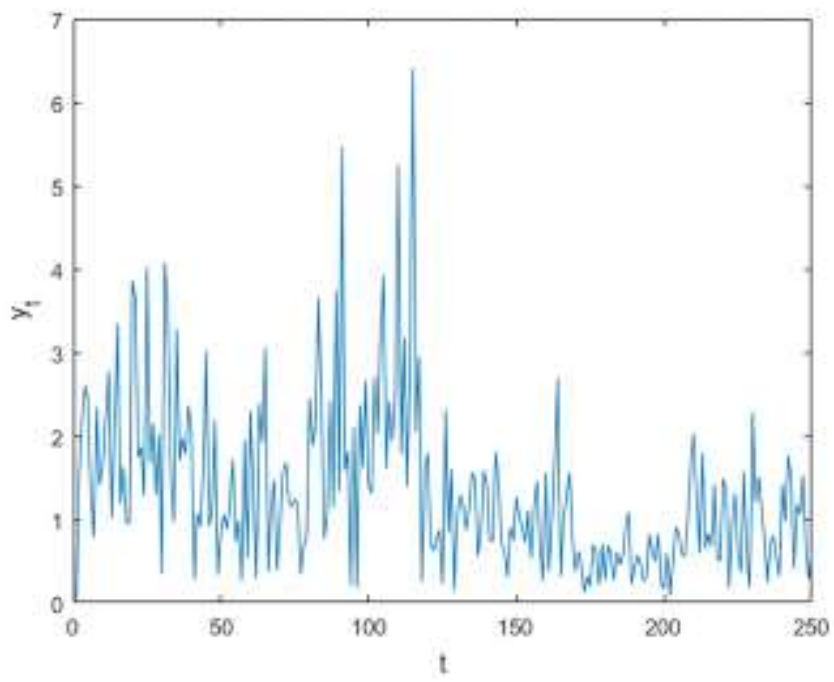

Fig. 4 Synthesis data with 5 change points

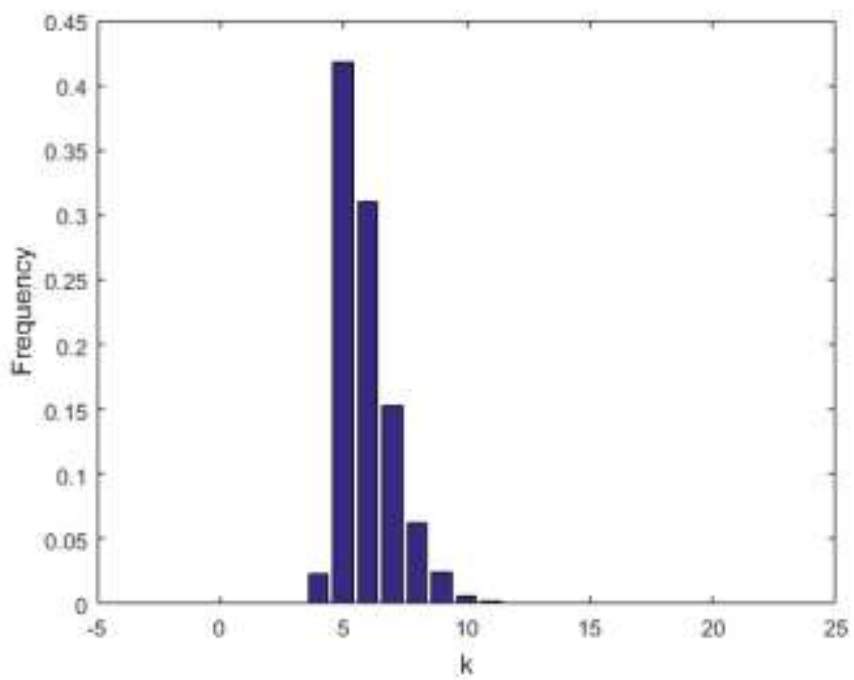

Fig. 5 Histogram of the number of change-points (second synthesis data) 


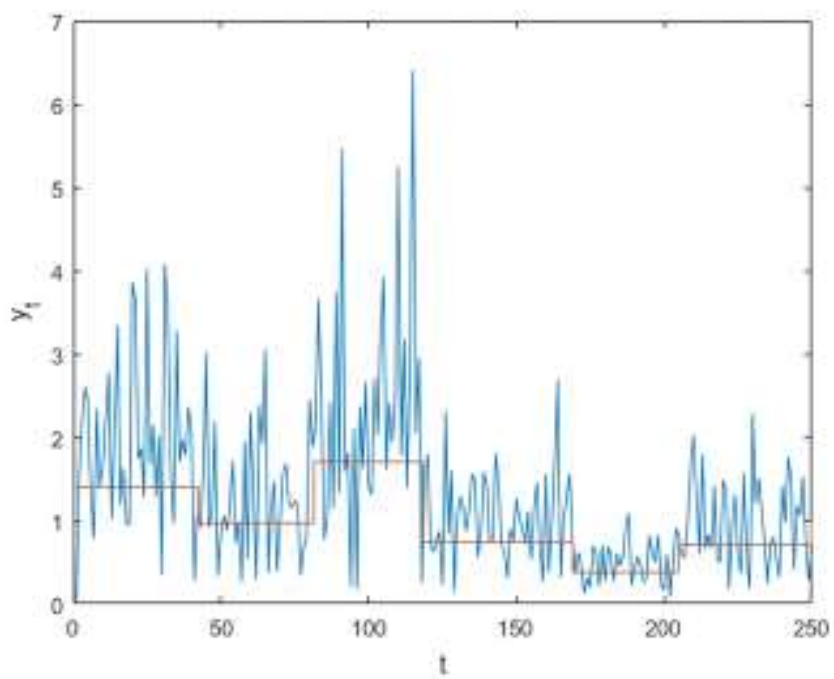

Fig. 6 Superposition between second synthesis data and parameter estimator

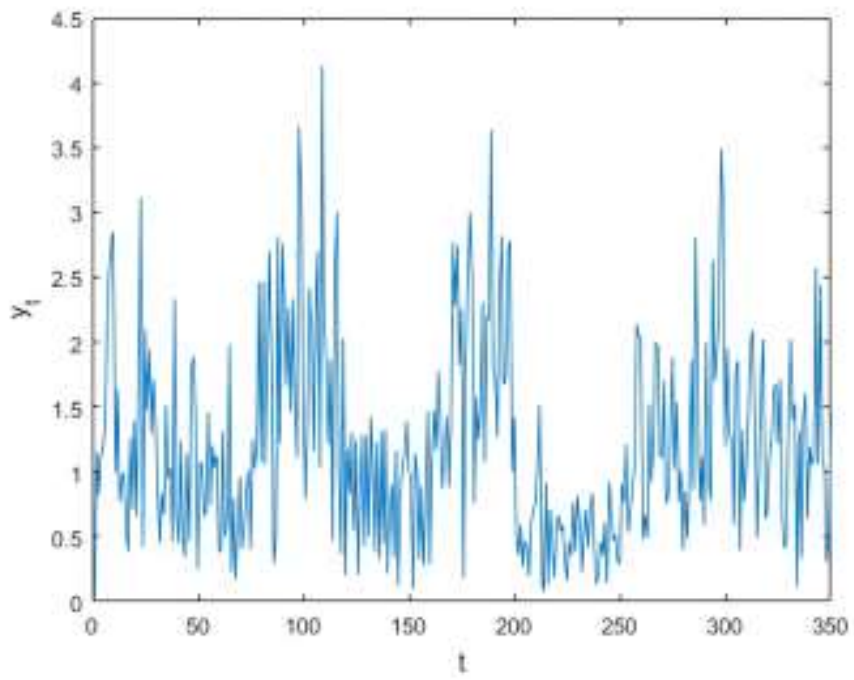

Fig. 7 Synthesis data with 6 change points

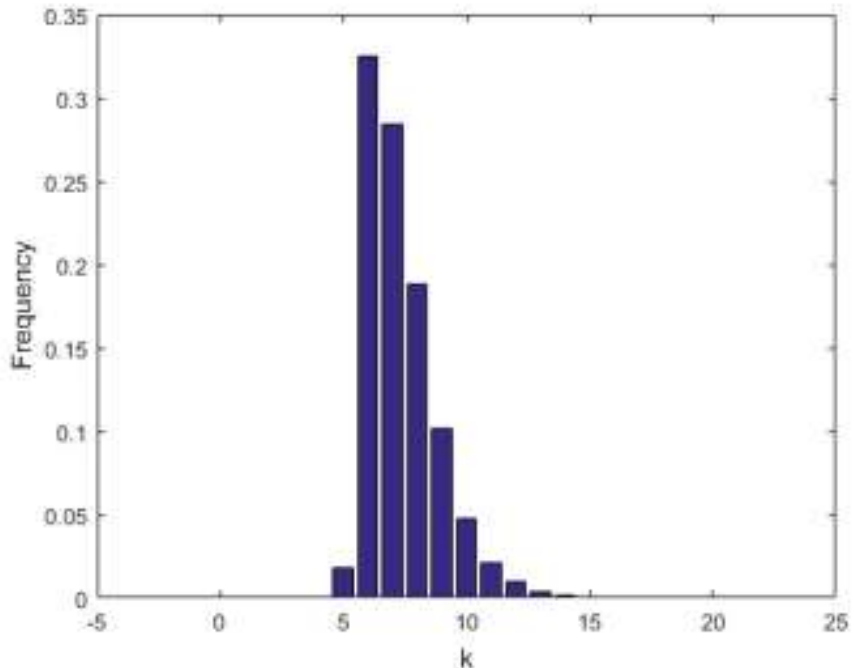

Fig. 8 Histogram of the number of change-points (third synthesis data) 


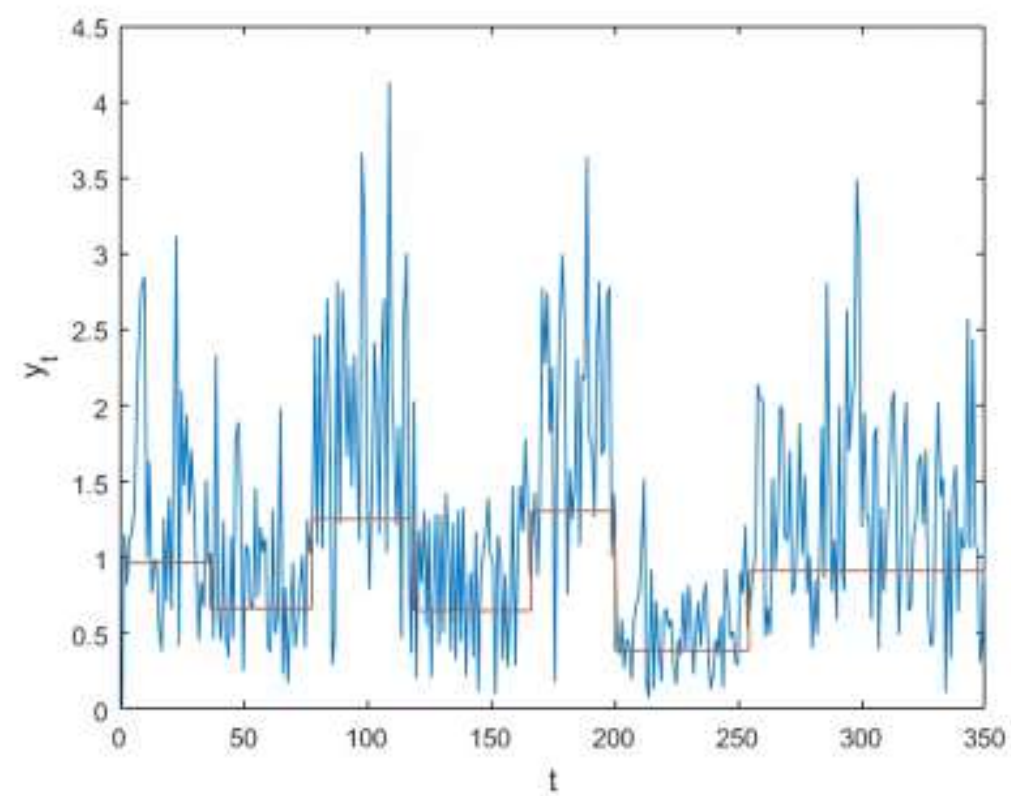

Fig. 9 Superposition between third synthesis data and parameter estimator

Table 2 The parameter estimator generated by the reversible jump MCMC algorithm

\begin{tabular}{ccc}
\hline$\hat{k}$ & $\hat{\imath}$ & $\hat{h}$ \\
\hline 4 & $(1,78,121,170,200,250)$ & $(1.1,1.7,0.9,0.2,0.9)$ \\
5 & $(1,43,81,118,169,205,250)$ & $(1.8,1.3,2.3,1.0,0.5,1.0)$ \\
6 & $(1,37,78,119,167,201,255,350)$ & $(1.4,0.9,1.8,0.9,1.8,0.6,1.3)$ \\
\hline
\end{tabular}

\subsection{Discussion}

This study has provided data modeling using piecewise constant model with Rayleigh noise. In addition, this study has also produced an algorithm to estimate the parameters of the piecewise constant model with Rayleigh noise. The first synthesis data (Fig. 1) was created using the parameter values in Table 1 (second row). In this study, $k_{\max }=20$ and $\alpha=0.20$. The synthesis data in Fig. 1 is modeled as a piecewise constant model with Rayleigh noise. Furthermore, this synthesis data has been used to rediscover the value of the model parameters. The model parameters have been estimated using the reversible jump MCMC algorithm. The parameter estimators that have been generated by this algorithm are presented in Table 2 (second row). When comparing the parameter estimators and parameter values, the parameter estimators in Table 2 (second row) are close to the parameter values in Table 1 (second row). The same results were also found for the second synthesis data (Fig. 4) and the third synthesis data (Fig. 7). This simulation study shows that the reversible jump MCMC algorithm can be used to estimate the model parameters.
If the results of this study are compared with previous studies. In [6-7], previous researchers used gamma noise. But in this study, the author uses noise with Rayleigh distribution. Thus, this article complements the results of previous studies. This article provides an alternative for modeling data that has Rayleigh noise. In terms of noise, the Rayleigh distribution is a special case of the Weibull distribution, so in general the research can be extended to the piecewise constant model with Weibull noise.

\section{CONCLUSIONS}

This research has studied the parameter estimation procedure of the piecewise constant model with Rayleigh noise based on the reversible jump MCMC algorithm. The results have shown that the reversible jump MCMC algorithm can simultaneously estimate the parameters of the piecewise constant model with Rayleigh noise. In addition, the research results can add to the literature related to signal modeling using piecewise constant models with Rayleigh noise. Further research can be extended to the estimation procedure of the 
piecewise constant with Weibull noise.

\section{ACKNOWLEDGMENTS}

The authors would like to thank the Ministry of Education, Culture, Research and Technology (Indonesia) for providing research grants with a basic research scheme. The authors would also like to thank the reviewers of International Journal of GEOMATE for their comments and suggestions to improve the quality of this article.

\section{REFERENCES}

[1] Beaulieu C., Chen J., and Sarmiento J.L., Change-point analysis as a tool to detect abrupt climate variations, Philos. Trans. R. Soc. A Math. Phys. Eng. Sci., Vol. 370, No. 1962, 2012, pp. 1228-1249.

[2] Sadia F., Boyd S., and Keith J.M., Bayesian change-point modeling with segmented ARMA model, PLoS One, Vol. 13, No. 12, 2018, pp. 1-23.

[3] Buscot M.-J., Wothersponn S.S., Magnussen C.G., Juonala M., Sabin, M.A., Burgner D. P., Lehtimaki T., Viikari J.S.A., HutriKahonen N., Raitakari O.T., and Thomson R.J., Bayesian hierarchical piecewise regression models: a tool to detect trajectory divergence between groups in long-term observational studies, BMC Med. Res. Methodol., Vol. 17, No. 1, pp. 1-16, 2017.

[4] Khoolenjani N.B. and Alamatsaz M.H., Extension of de Bruijn's identity to dependent non-Gaussian noise channels, J. Appl. Probab., Vol. 53, No. 2, pp. 360-368, 2016.

[5] Aubertt G. and Aujol J.F., A variational approach to removing multiplicative noise, SIAM J. Appl. Math., Vol. 68, No. 4, 2008, pp. 925-946.

[6] Suparman and Doisy M., Bayesian segmentation in signal with multiplicative noise using reversible jump MCMC, Telkomnika (Telecommunication Comput. Electron. Control., Vol. 16, No. 2, pp. 673680, 2018.

[7] Suparman, Bayesian estimation in piecewise constant model with gamma noise by using reversible jump MCMC, Math. Stat., Vol. 8, No. 2, 2020, pp. 17-22.

[8] Best D.J., Rayner J.C.W., and Thas O., Easily applied tests of fit for the Rayleigh distribution, Sankhyā Indian J. Stat. Ser. B, Vol. 72, No. 2, 2008, pp. 254-263.

[9] Dass S.C., Lim C.Y., Maiti T., and Zhang Z., Clustering curves based on change point analysis: a nonparametric Bayesian approach, Stat. Sin., Vol. 25, No. 2, 2015, pp. 677-708.

[10] Oliver C. and Quegan S., Understanding Synthetic Aperture Radar Images, SciTech, 2004.

[11] Yang C.-S. and Ouchi K., Application of Velocity Bunching Model to Estimate Wave Height of Ocean Waves using Multiple Synthetic Aperture Radar Data, J. Coast. Res., Vol. 79, No. 10079, 2017, pp. 94-98.

[12] Hu J., Zhao Z., Yalamanchili H.K., Wang J., Ye K., and Fan X., Bayesian detection of embryonic gene expression onset in $\mathrm{C}$. elegans, Ann. Appl. Stat., Vol. 9, No. 2, 2015, pp. 950-968.

[13] Green P.J., Reversible jump Markov chain Monte Carlo computation and Bayesian model determination, Biometrika, Vol. 82, No. 4, 1995, pp. 711-743.

[14] Heikkinen J. and Hogmander H., Fully Bayesian Approach to Image Restoration with an Application, Biogeography, 1994.

Copyright (C) Int. J. of GEOMATE All rights reserved, including making copies unless permission is obtained from the copyright proprietors. 